\title{
Preliminary Novel Analysis on Antimicrobial Properties of Concentrated Growth Factor against Bacteria-Induced Oral Diseases
}

\author{
Muhammad Syafiq Alauddin ${ }^{1}$ Nabilah Mohd Yusof ${ }^{2}$ \\ ${ }^{1}$ Department of Conservative Dentistry and Prosthodontics, Faculty \\ of Dentistry, Universiti Sains Islam Malaysia, Kuala Lumpur, Malaysia \\ ${ }^{2}$ Faculty of Dentistry, Universiti Sains Islam Malaysia, Kuala Lumpur, \\ Malaysia \\ 3 Department of Basic Sciences and Oral Biology, Faculty of Dentistry, \\ Universiti Sains Islam Malaysia, Kuala Lumpur, Malaysia
}

\author{
Aini Sufinah Adnan² Zulfahmi Said ${ }^{3}$
}

Eur J Dent 2022;16:901-910.

\author{
Address for correspondence Muhammad Syafiq Alauddin, BDS, \\ DClinDent, MProstho RCS (Edin), Department of Conservative \\ Dentistry and Prosthodontics, Universiti Sains Islam Malaysia, 55100 \\ Kuala Lumpur, Malaysia (e-mail: syafiq.alauddin@usim.edu.my).
}

\section{Abstract \\ Keywords \\ - antibiofilm \\ - concentrated growth factor \\ - oral pathogen \\ - platelet-rich concentrate \\ - antibacterial}

Objective Concentrated growth factor (CGF) is particularly gaining acceptance and popularity in regenerative dentistry. Nonetheless, there are no available studies showing its effect against microorganisms of oral cavity particularly in chronic oral disease-induced biofilms. This in vitro research was conducted to determine the antimicrobial effects of CGF against Staphylococcus aureus sp. (S. aureus) and Streptococcus mutans sp. (S. mutans).

Materials and Methods Blood samples were obtained from a healthy volunteer. CGF was then prepared using specialized centrifugation equipment (Medifuge, Silfradent, Santa Sofia FC, Italy) and protocol. Antimicrobial activity of the CGF was observed and recorded on standard strains of $S$. aureus and $S$. mutans using a well diffusion method to determine the inhibition zone, broth microdilution to determine minimum inhibitory concentration (MIC) and minimum bactericidal concentration (MBC), and crystal violet assay for biofilm assessment, with chlorhexidine (CHX) $0.12 \%$ used as a positive control. Statistical analysis was then performed using one-way analysis of variance followed by Tukey Test post hoc analysis.

Results It was observed that there was a presence of clear zones of inhibition around the CGF after 24 hours of incubation. The mean diameter of the inhibition zone was $1.26 \pm 0.12 \mathrm{~nm}$ and $1.20 \pm 0.06 \mathrm{~nm}$ for $S$. aureus and $S$. mutans, respectively, with significance difference $(p<0.05)$ against the control group CHX $0.12 \%$. The MIC values of the CGF against S. aureus and S. mutans were $47.9 \%$ and $34.17 \%$, respectively, and the MBC values of the CGF against S. aureus and S. mutans were $100 \%$. The viability and the ability in inhibiting the biofilm formation of $S$. mutans and $S$. aureus following treatment with CGF showed a reduction in the concentration-dependent manner as compared with the control group.

Conclusion CGF possesses antimicrobial and antibiofilm activity against $S$. aureus and S. mutans.
DOI https://doi.org/ $10.1055 / \mathrm{s}-0041-1742121$. ISSN 1305-7456. (c) 2022. The Author(s).

This is an open access article published by Thieme under the terms of the Creative Commons Attribution License, permitting unrestricted use, distribution, and reproduction so long as the original work is properly cited. (https://creativecommons.org/licenses/by/4.0/)

Thieme Medical and Scientific Publishers Pvt. Ltd., A-12, 2nd Floor, Sector 2, Noida-201301 UP, India 


\section{Introduction}

Growth factors are bioactive proteins that control the woundhealing process. One of the initial uses of platelet concentrates in dentistry includes the innovation of fibrin glues used mainly to promote healing by promotion of healing and wounds protection. ${ }^{1}$ Platelet concentrates are retrieved from the blood extract and then later developed to promote regeneration of cell by stimulating its growth factor and cell proliferation at the surgical site. Platelets contain enormous volume of key growth factors that include bone morphogenetic protein (BMP), platelet-derived growth factor (PDGF), insulin-like growth factor (IGF), vascular endothelial growth factor (VEGF), transforming growth factor (TGF)- $\beta 1$, and TGF- $\beta 2{ }^{2,3}$ The first generation of platelet concentrates is platelet-rich plasma (PRP), introduced by Marx in $1988 .^{2}$ It was initially developed for medical purposes for its ability in retaining growth factors and promoting better healing by merging the effect of growth factors and properties of fibrin sealant, thus, giving the ideal delivery system of growth factors. ${ }^{4}$ PRP has been clinically applied in the procedures of sinus lift and ridge augmentation, socket preservation, alveolar cleft palate repair, and soft tissue procedures such as gingival grafts. ${ }^{5}$ The second generation of platelet concentrates consists of platelet-rich fibrin (PRF) and concentrated growth factor (CGF). PRF was developed by Choukroun et al in 2001, produced by centrifuging venous blood, taken without biochemical modification. As a result, a solid fibrin clot is formed that is rich in platelets, leukocytes, and growth factors. ${ }^{3}$ Another second-generation platelet concentrate, which is known as concentrated growth factor, developed by Sacco et al, was produced by centrifuging venous blood with its centrifugal technique in a manner similar to PRF, but differed in the specific protocol of centrifugation speed utilizing a specific centrifugation machine. ${ }^{6}$ As a result, a harder fibrin structure with richer growth factors was produced compared with the typical platelet concentrates. CGF showed superiority in terms of tensile strength, growth factors, viscosity, and adhesive strength in comparison with the other platelet concentrates. ${ }^{6}$ CGF promotes angiogenesis, cell proliferation, matrix remodeling, and regeneration as it contains platelets, leukocytes, CD34+ stem cells, and various growth factors such as PDGF, TGF- $\beta 1$, epidermal growth factor (EGF), fibroblast growth factor (FGF), IGF-I, as well as VEGF. ${ }^{6,7}$ In clinical application, it was proven to be effective in hard tissue regeneration, sinus lift, osteonecrosis, and several other various oral surgery intervention and treatment. ${ }^{8-12}$ In a study reported by Pirpir et al, CGF had positive effects on implant stability and accelerated osseointegration. ${ }^{13}$

The pathogenicity of oral Staphylococcus and Streptococci groups remains a pressing challenge for potential intraoral biofilm infection. Streptococcus mutans sp. (S. mutans) is a Gram-positive facultative anaerobe and for decades, it has been widely known as the etiological agents of dental caries. Streptococci species are the established early colonizers of salivary pellicle-coated oral surfaces and the most important virulence factor of this species is its biofilm formation ability, which is also known as the dental plaque, with firm adherence to the tooth surfaces. Staphylococcus aureus sp. (S. aureus) on the other hand is a Gram-positive, nonsporadic forming, spherical, and aerobic and facultative anaerobic bacterium belonging to the Staphylococcus genus. ${ }^{14,15}$ S. aureus has been acknowledged as a part of oral flora despite its isolation rates in the oral cavity associated with conditions such as angular cheilitis, suppurative parotitis, denture stomatitis, and acute dentoalveolar infections. ${ }^{16}$ In recent studies, it is also found that S. aureus may contribute to dental implant failure. ${ }^{17}$

There was a plethora of available literature indicating the potential antimicrobial and antibacterial properties of the platelet concentrates especially PRF and its derivatives including i-PRF, H-PRF, and L-PRF. ${ }^{18-21}$ To the best of the authors' knowledge, there is no available literature on antimicrobial properties of CGF against microorganisms to date as no study has been done on this matter. ${ }^{22}$ Hence, this study was aimed to investigate the potential antimicrobial properties of CGF on oral Staphylococcus (S. aureus) and Streptococcus (S. mutans) isolates.

\section{Materials and Methods}

\section{Blood Collection and CGF Preparation}

Institution ethical approval was achieved for this study (USIM/JKEP/2020-100). Blood samples were collected from a healthy donor. Verbal and informed information was communicated to the subject followed by the signed consent form. The subject was systemically healthy, a nonsmoker, not pregnant, no history of blood disorder, without symptoms of infection, and was not on antibiotics for the past 3 months prior to this study. A total volume of $20 \mathrm{~mL}$ of venous blood was collected by venipuncture in two $10 \mathrm{~mL}$ tubes without anticoagulants. The blood samples were centrifuged using a specific program at 30 seconds for acceleration, $2,700 \mathrm{rpm}$ for 2 minutes, $2,400 \mathrm{rpm}$ for 4 minutes, $2,700 \mathrm{rpm}$ for 4 minutes, 3,000 rpm for 3 minutes, and finally with deceleration for 36 seconds using a specialized machine, Medifuge MF200 (Silfradent, Italy; - Fig. 1). The centrifuged blood resulted in three layers: the uppermost layer of blood serum, the middle layer of fibrin buffy coat, and the bottom layer of red blood cells (RBC). The fibrin buffy CGF clot was removed from the tube using sterile tweezers and then separated from the RBC base using sterile microsurgical scissors as depicted in - Fig. 2. The CGF coat from one tube was compressed and the liquefied CGF (L-CGF) was then collected. The other CGF coat was cut into smaller fragments using the microsurgical scissors to act as the CGF gel (G-CGF). The samples were analyzed immediately prior to the gelation occurrence.

\section{Microorganisms Preparation}

S. aureus sp. and S. mutans sp. were used in this study. The pure bacterial cultures were then isolated to prepare working cultures. Bacterial isolation in agar plate was performed using the streak plate isolation method by separating the bacteria into four quadrants using a heated inoculating loop. The brain heart infusion (BHI) broth solution was mixed with the pure cultures respectively using a heated inoculating loop. S. aureus and S. mutans working cultures were then incubated at $37^{\circ} \mathrm{C}$ for 24 hours. 


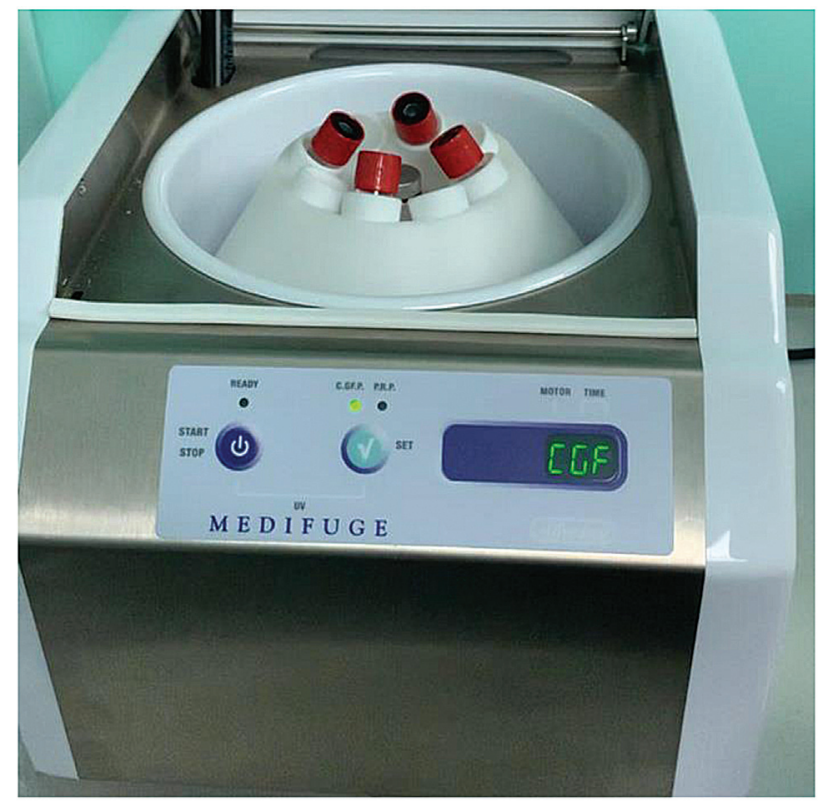

Fig. 1 Medifuge MF200 (Silfradent, Italy) with specific program at 30 seconds for acceleration, 2,700 rpm for 2 minutes, 2,400 rpm for 4 minutes, 2,700 rpm for 4 minutes, 3,000 rpm for 3 minutes, and finally with deceleration for 36 seconds.
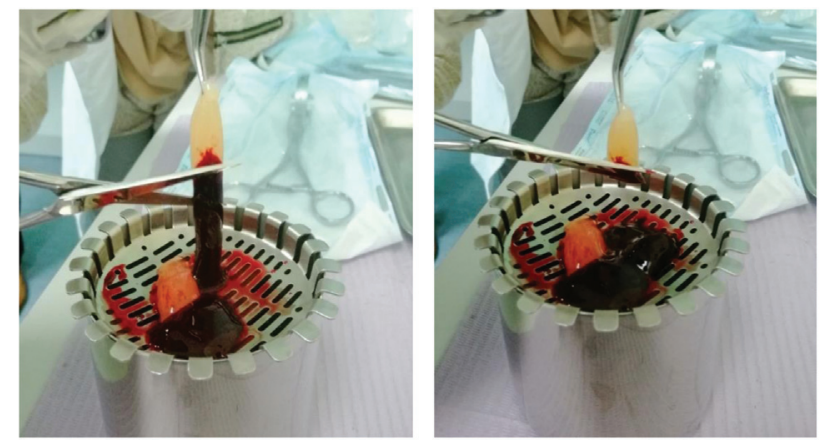

Fig. 2 Separation of concentrated growth factor from red blood cell base.

\section{Determination of Zone of Inhibition}

The cloudiness of the bacterial suspension in the working broth was then adjusted with a dilution using micropipette in accordance with the 0.5 and 1.0 McFarland's standard solution for $S$. aureus and $S$. mutans respectively. The prepared CGF was screened for its antimicrobial properties using the disk diffusion method. The spread plate method was performed by dipping a sterile cotton swab into the S. aureus working broth and then swabbed onto the BHI agar plate. The prepared agar plates were then divided into three sections, G-CGF, L-CGF, and positive control chlorhexidine (CHX) $0.12 \%$ solution, and were labeled accordingly as in -Fig. 3. An autoclaved, sterile filter paper disc of $6 \mathrm{~mm}$ in diameter was held using a pair of sterile forceps and placed onto the section of the agar accordingly. Then $7 \mu \mathrm{L}$ of $\mathrm{CHX}$ solution was loaded and micropipette onto the paper disc of the CHX section and was repeated for the L-CGF. The G-CGF was placed onto the section using sterile tweezers. The same procedure was repeated for $S$. mutans as well. The plates were

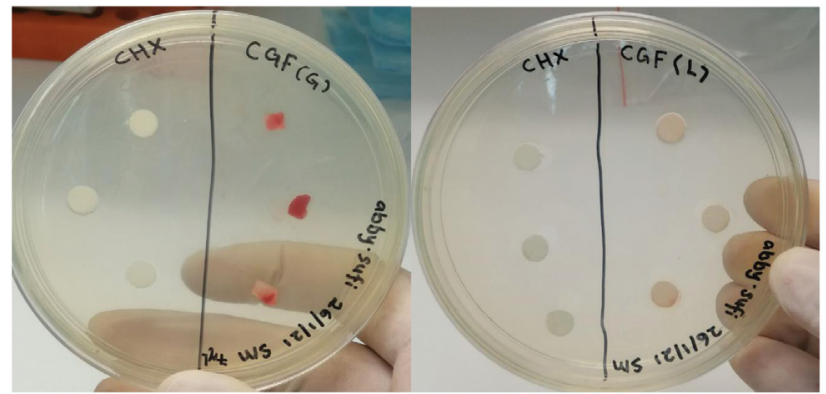

Fig. 3 An example depicting inoculation of concentrated growth factor gel and liquefied concentrated growth factor with Streptococcus mutans.

then incubated at $37^{\circ} \mathrm{C}$ for 24 hours. After 24 hours of incubation, the diameter of the zones of inhibition was measured thrice using a ruler and the average measurement was obtained. The growth of the inhibition zone was then measured and the average diameter of the inhibition zone of the three sections for each culture was recorded.

\section{Minimum Inhibitory Concentration (MIC) and Minimum Bactericidal Concentration (MBC)}

To determine the minimum inhibitory concentration (MIC) and minimum bactericidal concentration (MBC), the broth microdilution method was utilized to assess the antibacterial activity of the CGF. An MIC assay was performed onto the 96well plates using a micropipette to determine the bacterial culture and L-CGF according to its concentration ranging from 100 to $0 \%$. Negative control well was the untreated cell (BHI broth) alone while the positive control well contained cells treated with CHX and the procedure was done in a triplicate manner. After 24 hours of incubation, the plates were placed into the microplate reader to obtain the reading for MIC. The workflow is depicted in - Fig. 4. An MBC test was performed using a micropipette of $5 \mu \mathrm{L}$ from the MIC wells that exhibited no bacterial growth onto the cultured plates and incubated overnight at $37^{\circ} \mathrm{C}$. After 24 hours incubation period, the formation of colonies was recorded.

\section{Viability Testing}

Viability testing was done to assess the growth pattern of both bacteria following the treatment with CGF. The test was performed by adding 3-(4,5-dimethylthiazol-2-yl)-2,5diphenyl tetrazolium bromide reagent into the remaining mixture of the 96 wells. The plate was then incubated for 30 minutes. The change in color of the mixture from yellow to purple was observed. The increment in purple resulted from the increasing number of cells recorded. The absorbance was then measured in a microplate reader at $540 \mathrm{~nm}$. The workflow of the viability testing is as per - Fig. 5 .

\section{Biofilm Formation Assay}

A biofilm formation assay was performed to investigate the effect of the CGF treatment against the biofilm formation. A biofilm formation assay was done by placing diluted bacterial culture into the wells followed with the addition of CGF with a negative control well containing untreated cells and a 


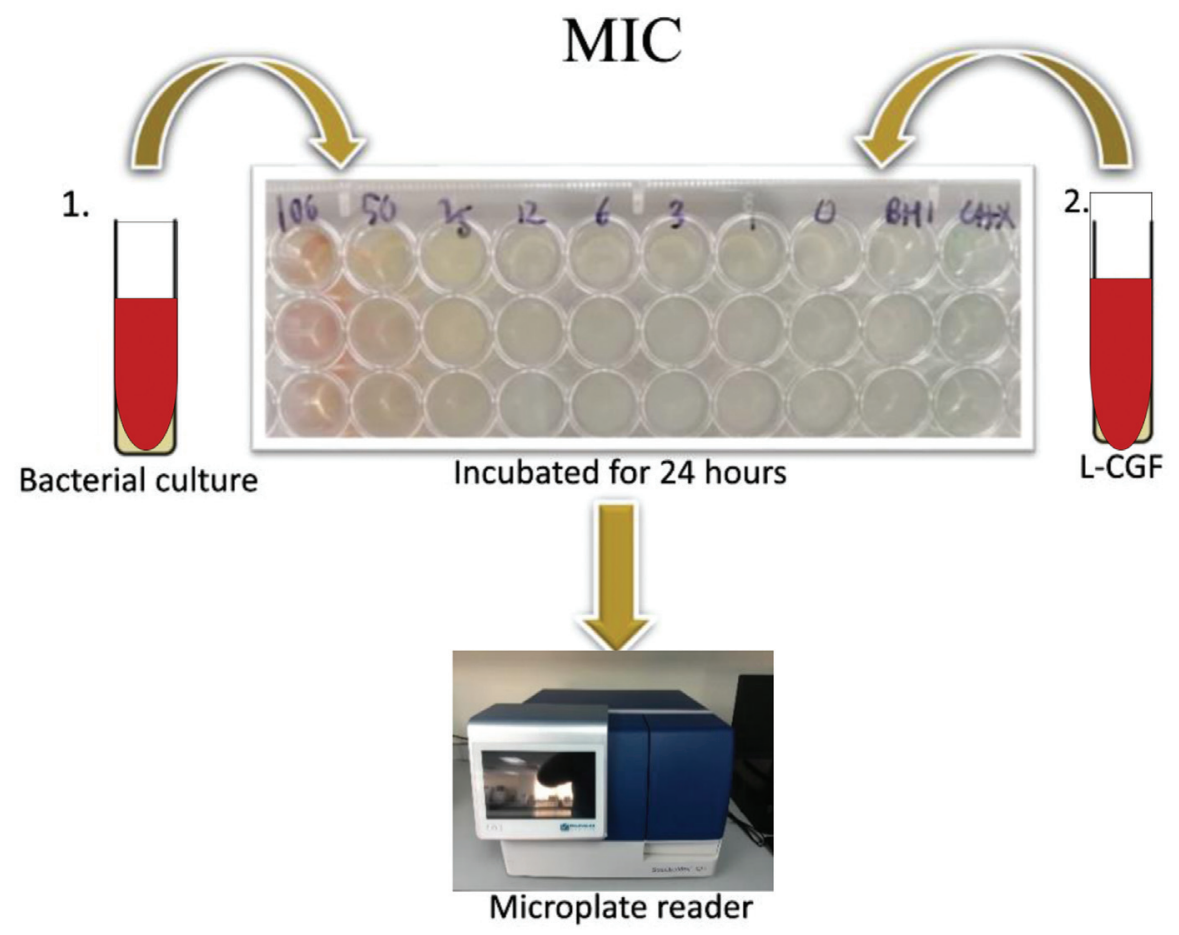

Fig. 4 The workflow for Minimum Inhibitory Concentration (MIC) protocol.

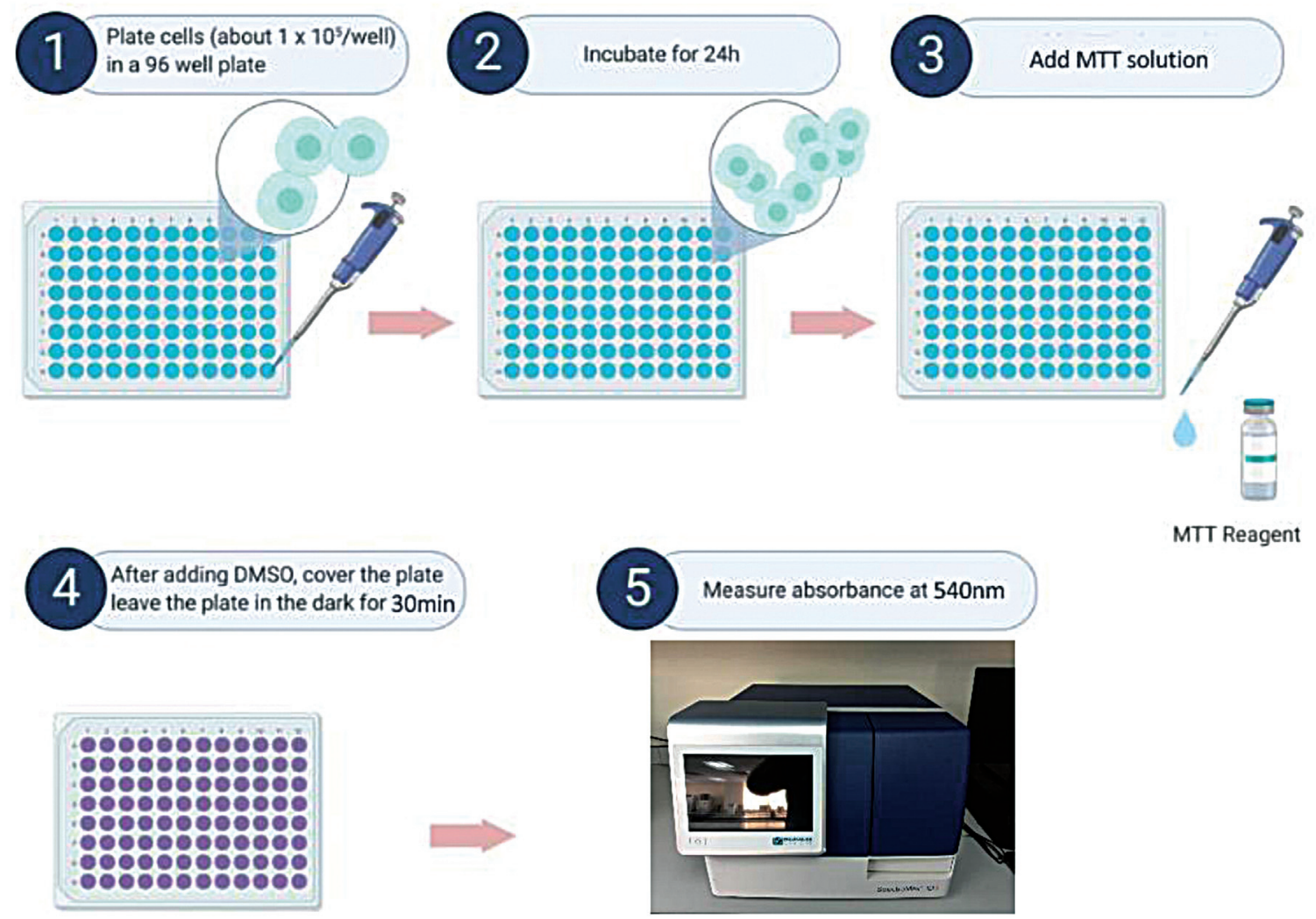

Fig. 5 The workflow for cell viability testing protocol. 


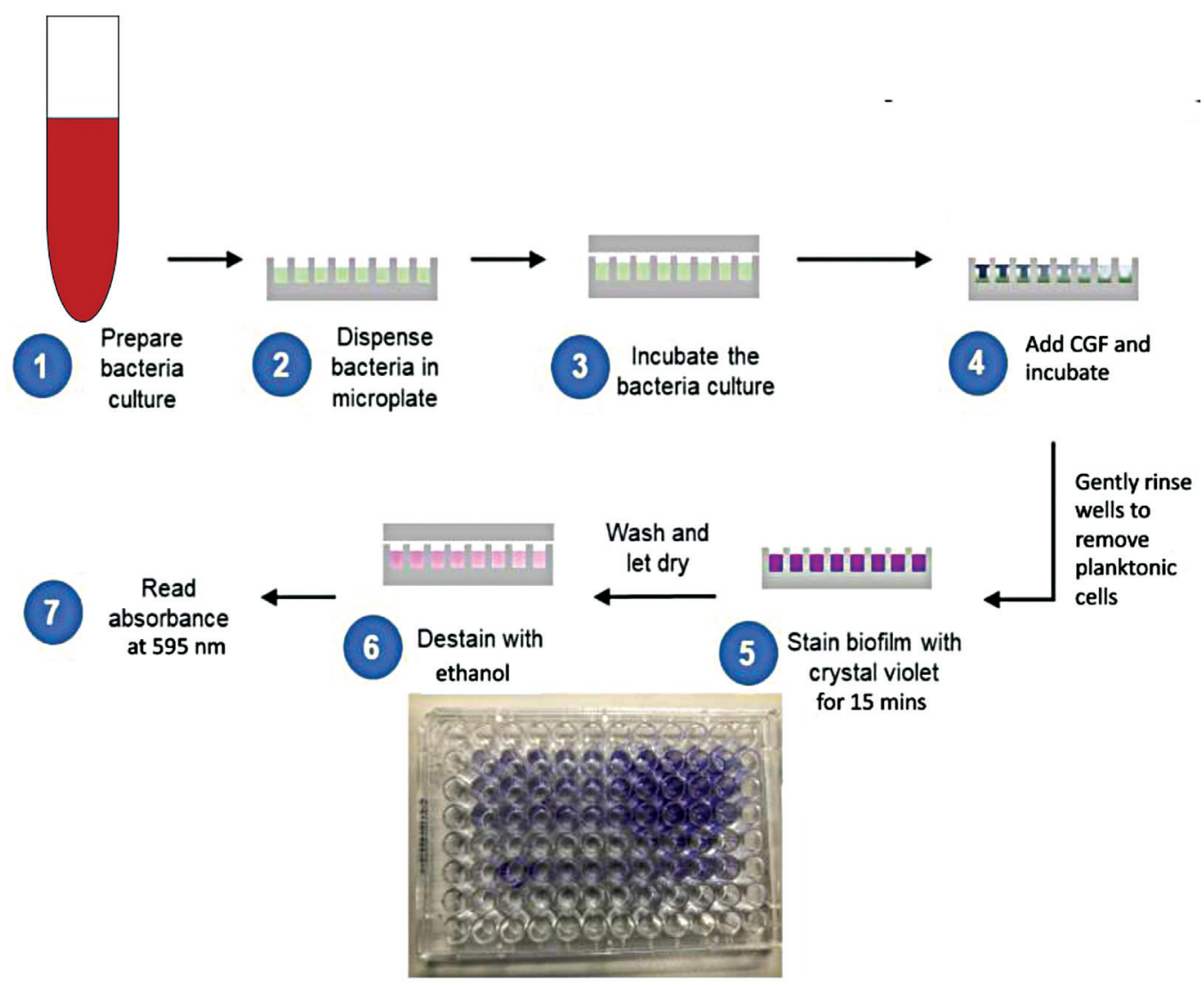

Fig. 6 The workflow of biofilm formation assay protocol.

positive control well containing cells treated with $\mathrm{CHX}$. The plate was then inoculated and incubated, later decanted, and gently washed twice. The adherent bacteria were then stained using a crystal violet dye and then gently washed twice. The bound dye was extracted from the stained cells using ethanol. The biofilm formation was then quantified by measuring the absorbance of the solution at $595 \mathrm{~nm}$ in a microplate reader. The workflow of the entire protocol is depicted in - Fig. 6 .

\section{Statistical Analysis}

The data obtained from this study were further analyzed using the Statistical Package for the Social Sciences, version 21.0 software(IBM SPSS; IBM Corporation, New York, United States).
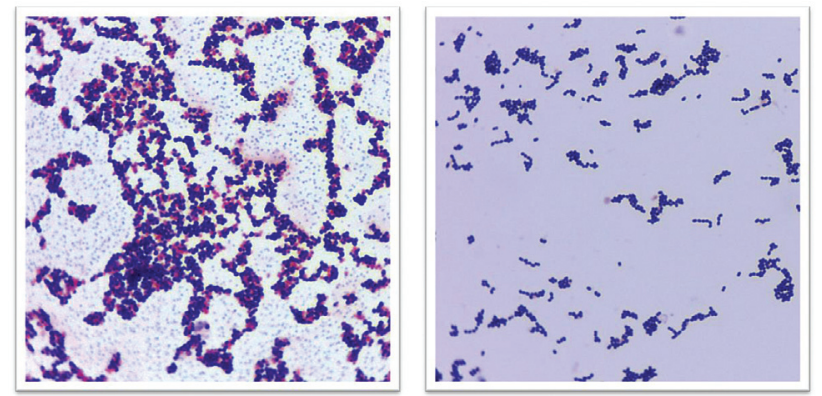

Fig. 7 Streptococcus mutans (left) and Staphylococcus aureus (right) under 100x magnification.
A normality distribution was performed initially and further one-way analysis of variance was used. Tukey Test post hoc analysis was performed to analyze which group was the one that gave the significant difference. A $p$-value with less than 0.05 was considered as statistically significant with the sample set at $95 \%$ of confidence interval. The data were expressed in mean \pm standard deviation.

\section{Results}

\section{Bacteria Morphological Analysis}

The morphology of $S$. aureus and $S$. mutans was observed under the image analyzer at 100x magnification as per - Fig. 7 .

\section{Determination of Zone of Inhibition}

Clear zones of inhibition were observed around CHX, L-CGF, and G-CGF against $S$. aureus and S. mutans after 24-hour incubation as per $\mathbf{- F i g . ~ 8}$. The diameter of the clear zones was measured using triplicate readings, and the results are as tabulated in -Table 1.

\section{Determination of Antimicrobial Activity}

The antibacterial activity of the CGF was measured using the MIC and MBC assessments. The value of the MIC was measured using a microplate reader with an optical density at $540 \mathrm{~nm}$. The MIC value of the CGF against $S$. aureus and S. mutans was $47.85 \%$ and $35.59 \%$, respectively. The MBC 

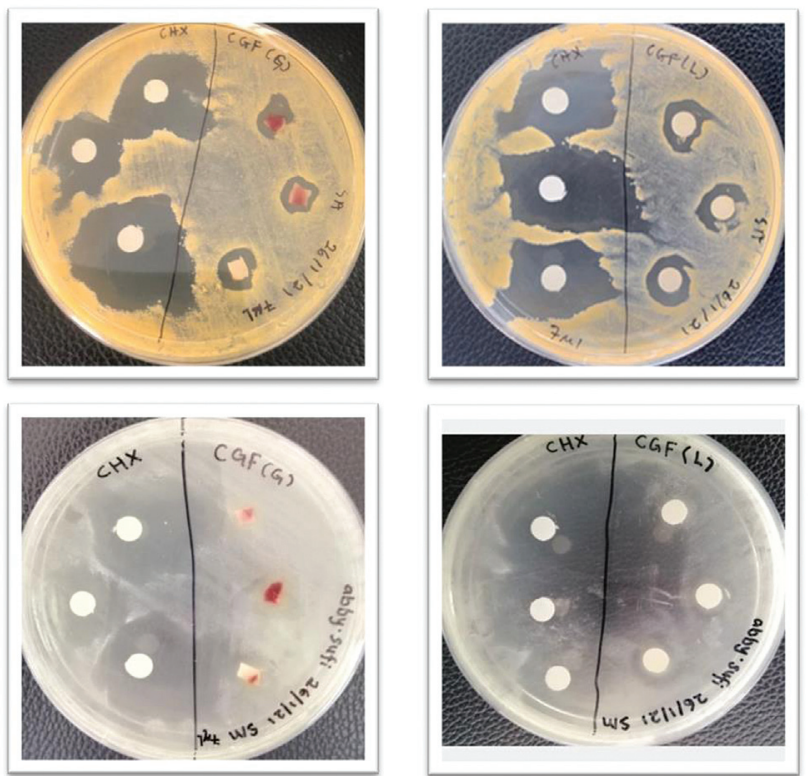

Fig. 8 Determination of zone of inhibition showed that both concentrated growth factor gel and liquefied concentrated growth factor produced clear zone of inhibition in comparison to chlorhexidine $0.12 \%$ (positive control).

Table 1 Mean values and standard deviation for the diameter of zones of inhibition

\begin{tabular}{|l|l|l|l|}
\hline Organism & \multicolumn{4}{|l|}{ Mean \pm SD (cm) } & $\begin{array}{l}\text { CHX } \\
\text { (positive } \\
\text { control) }\end{array}$ \\
\hline & G-CGF & L-CGF & $2.51 \pm 0.32$ \\
\hline $\begin{array}{l}\text { Staphylococcus } \\
\text { aureus }\end{array}$ & $0.92 \pm 0.10^{*}$ & $1.26 \pm 0.12^{*}$ & $2.83 \pm 0.36$ \\
\hline $\begin{array}{l}\text { Streptococcus } \\
\text { mutans }\end{array}$ & $1.27 \pm 0.23^{*}$ & $1.20 \pm 0.06^{*}$ & \\
\hline
\end{tabular}

Abbreviations: CHX, chlorhexidine (0.12\%); CGF-G, concentrated growth factor gel; CGF-L, liquefied concentrated growth factor; SD, standard deviation.

Note: There is significant difference of G-CGF and L-GCF compared with $\mathrm{CHX}$ in both $\mathrm{S}$. aureus and $\mathrm{S}$. mutans. ${ }^{*} p<0.05$.

value of the CGF against $S$. aureus and S. mutans was determined at $100 \%$. If the $\mathrm{MBC} / \mathrm{MIC}$ ratio is $\leq 4$, it was considered as bactericidal while $>4$ is considered as bacteriostatic. The value of the MBC/MIC ratio of the CGF is 2 and the results are tabulated as showed in - Table 2.

\section{Viability Analysis}

Inhibition of the $S$. mutans and $S$. aureus growth was observed in a concentration-dependent manner following

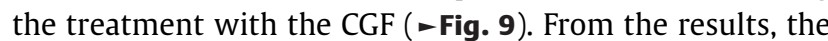
CGF at a $100 \%$ concentration showed comparable/similar efficacy in inhibiting the growth of both bacteria compared with the CHX ( $p>0.05)$ while other concentrations of the CGF have shown less efficacy in inhibiting both bacteria as compared with the control group $(p<0.05)$.
Table 2 Determination of antimicrobial activity of concentrated growth factor

\begin{tabular}{|l|l|l|l|}
\hline Organism & MIC (\% v/v) & $\begin{array}{l}\text { MBC } \\
(\% \mathrm{v} / \mathrm{v})\end{array}$ & $\begin{array}{l}\mathrm{MBC} / \mathrm{MIC} \\
\text { ratio }\end{array}$ \\
\hline $\begin{array}{l}\text { Staphylococcus } \\
\text { aureus }\end{array}$ & $47.85 \pm 4.75$ & 100 & $2(+)$ \\
\hline $\begin{array}{l}\text { Streptococcus } \\
\text { mutans }\end{array}$ & $35.59 \pm 2.04$ & 100 & $2(+)$ \\
\hline
\end{tabular}

Abbreviations: MBC, minimum bactericidal concentration; MIC, minimum inhibitory concentration.
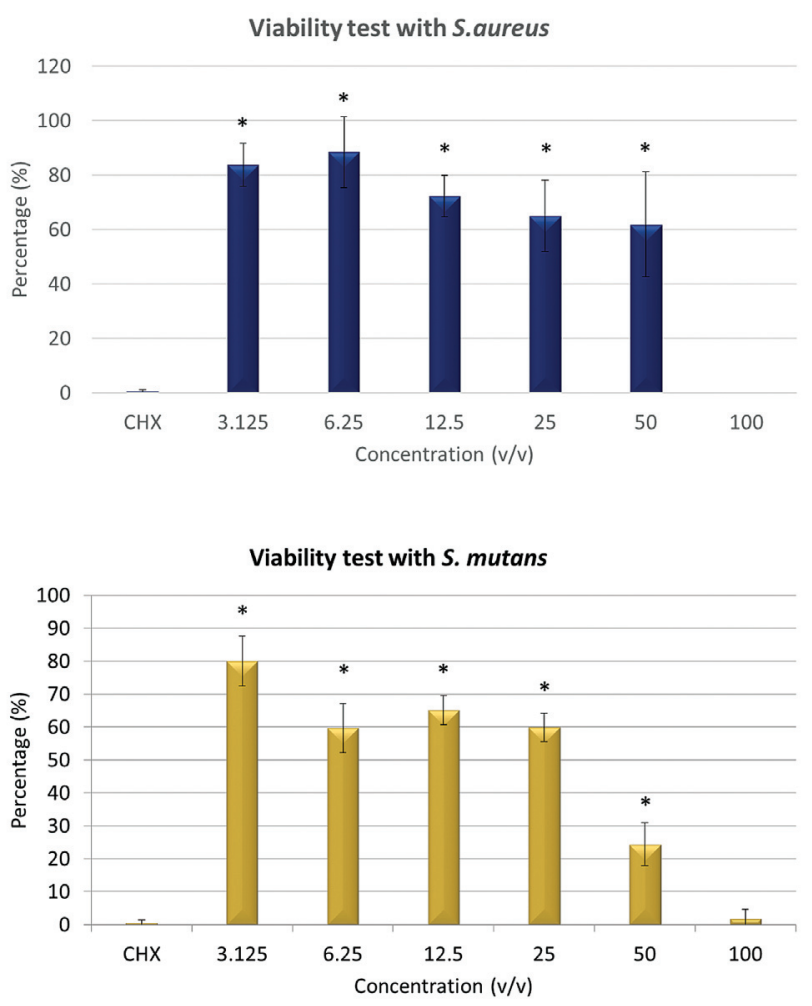

Fig. 9 Reduction in overall cell viability of Streptococcus mutans and Staphylococcus aureus in concentration-dependent manner following treatment with concentrated growth factor with $\left({ }^{*}\right)$ showed significant difference with chlorhexidine $0.12 \%(p<0.05)$.

\section{Biofilm Inhibition}

The investigation of the biofilm assay revealed that the inhibition of the biofilm produced by $S$. mutans and $S$. aureus was observed as the concentrations of the CGF increased, as depicted in - Fig. 10. Antibiofilm activity of the CHX against both bacterial strains was also plotted. It showed that the antibiofilm activity of the CGF against $S$. aureus was insignificant $(p>0.05)$, particularly at $1-50 \%$ concentration as compared with the $\mathrm{CHX}$ as the positive control. At a $100 \%$ higher concentration, the inhibition of the biofilm formation of the CGF in comparison to the CHX $(p<0.05)$ clearly shows that the CGF is more effective as an antibiofilm agent as compared with the CHX for $S$. aureus. Nonetheless, there was no significant difference $(p>0.05)$ between the CGF at all concentrations as compared with the CHX against the biofilm produced by S. mutans (-Fig. 11). 


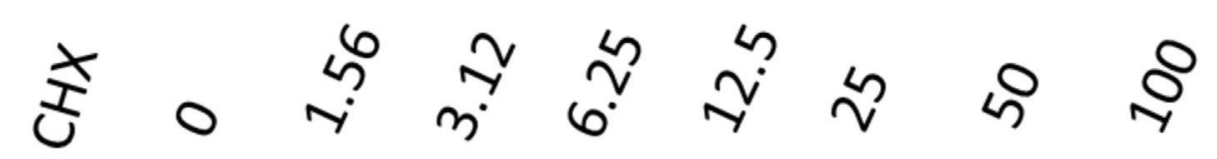

\section{S. aureus}
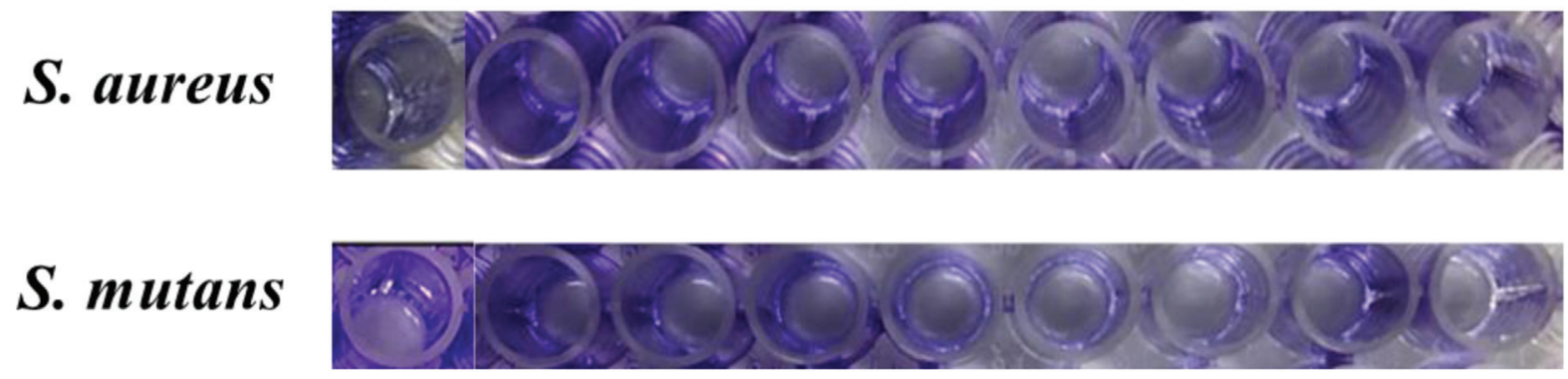

Fig. 10 Inhibition of biofilm was observed as the concentration of concentrated growth factor increases.

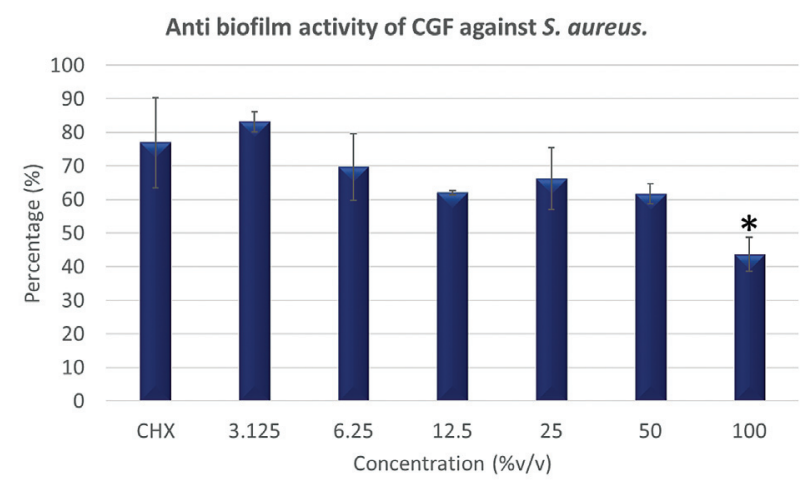

Anti biofilm activity of CGF against $S$. mutans.

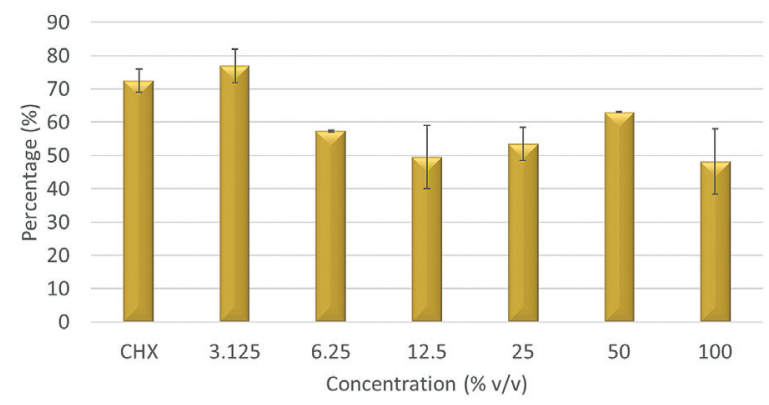

Fig. 11 Reduction in capability of concentrated growth factor (CGF) in inhibiting biofilm formation of Streptococcus mutans and Staphylococcus aureus in concentration-dependent manner following treatment with CGF with $\left(^{*}\right)$ showed significant difference with chlorhexidine $0.12 \%(p<0.05)$.

\section{Discussion}

There is a pressing need for the control and inhibition of oral biofilm that is responsible for the formation of dental caries. The pathogenicity of the dental biofilm particularly with the presence and formation of microorganisms within the extracellular matrix will only increase the resistance toward the host immune system and other various antibacterial agents. ${ }^{23} \mathrm{~S}$. mutans is a primary etiologic agent of human dental caries and is particularly effective at forming biofilms on the hard tissues of the human oral cavity. Adherence of S. mutans to dental surfaces is the first step in the formation of biofilms by this organism. It was believed to be the primary etiologic agent for the formation and the development of mature dental biofilm that can lead to dental caries. ${ }^{24}$ On the other hand, the Staphylococcus infections at surgical sites remain one of the widespread postoperative complications. The Staphylococci attached to the wound surface is able to proliferate and produce a biofilm. It has been recently recognized that biofilms that produce bacterial infection are prevalent in postoperative wounds and are a causative factor in wound chronicity. ${ }^{25,26}$ In addition, S. aureus is associated with multiple chronic orofacial and intraoral infections and conditions including facial cellulitis, facial bullous infection, angular cheilitis, dentoalveolar abscess, and dry mouth. ${ }^{25-28}$ The formation of a Staphylococci biofilm matrix at the infection site may cause resistance toward treatment by impairing the immune response of the host and may further resist the action of antimicrobial agent. ${ }^{29}$ There was also notable prevalence with regard to the resistant strains of $S$. aureus including the methicillinresistant S. aureus that will prolong the hospital care and eventually lead to the impairment of the quality of care to the patients, given their opportunistic and highly communicative nature. ${ }^{16,30,31}$ It is paramount to prevent and treat the acquired infection from both microorganisms as early as possible to eliminate and subsequently prevent further advanced chronic oral-related diseases.

Platelet concentrates have been progressively developed due to their regenerative potential and anti-inflammatory properties. In dental procedures, the utilization of platelet concentrates aids in periodontal reconstruction, implant placement, periodontal surgeries, and regeneration. As such, autologous platelet concentrates are widely used and are particularly gaining popularity recently. ${ }^{7,32,33}$ Specifically, platelet concentrates such as the PRF is used in treatments of intrabony defects, extraction sockets and recession management, alveolar ridge and sinus floor augmentation, and bone regeneration involving dental implants. ${ }^{5}$ The CGF is a platelet concentrate with fibrin network consisting of platelets, leukocytes, and growth factors. One of the main advantages of 
the CGF is operatively it is easier to be handled and maintained with regard to the desired shape in accordance with the surgical application in comparison to other types of platelet concentrates. $^{34}$ This is due to its high cohesiveness that protects it from plasmin degradation, thus giving it a higher fibrin tensile strength and stability. ${ }^{35}$ The CGF is similar to the PRF in terms of no bovine thrombin or anticoagulant usage, which in turns reduces the risk associated with the use of additives. ${ }^{6}$ Nonetheless, there were differences detected such as when the overall centrifugation protocol and preparation done using different speeds of centrifugation and times, which resulted in a larger, high-density fibrin matrix, richer in growth factors. ${ }^{6}$ The usage of the peripheral layer in regenerative procedures is recommended ${ }^{6,35}$ due to various growth factors found in the CGF including PDGF, TGF- $\beta$, IGF, EGF, FGF, and BMP and supported additionally from the histological perspectives due to specific presence of platelets in the peripheral layer and specific constitution of the fibrin network. Nonetheless, the potential antimicrobial and antibiofilm activities of the CGF are relatively unknown due to lack of evidence in the body of literatures. Therefore, in this study the main aim is to investigate in vitro antimicrobial and antibiofilm activities of the CGF and compare it with the conventional $0.12 \%$ CHX against two groups of oral pathogenic, Grampositive facultative anaerobe represented by $S$. mutans and S. aureus.

In spite of the widespread interest and preference by the practitioners to use both the gel and liquid forms of platelet concentrate for regenerative and implant therapies, there were extremely limited data in the body of the literature on the utilization and benefits of the liquid form of platelet concentrates specifically for CGF in this study. ${ }^{36}$ In the present study, the present forms of the CGF, the G-CGF and L-CGF, were retrieved after the completed centrifugation protocol. The L-CGF was specifically obtained by compressing the CGF membrane using pliers (Silfradent, Sofia, Italy) and the liquid was then collected using a grid and dappen for fibrin separator (Silfradent, Sofia, Italy) accordingly. The results showed that both the G-CGF and L-CGF showed a distinct zone of inhibition on both $S$. mutans and $S$. aureus, where most of the inhibition zone was by the G-CGF on S. mutans $(1.27 \mathrm{~cm} \pm 0.23)$ and the minimum amount of inhibition zone was by the G-CGF on $S$. aureus $(0.92 \mathrm{~cm}$ $\pm 0.10)$. The maximum inhibition zone for both microorganisms was recorded by the positive control of CHX $0.12 \%$, which was established as a broad-spectrum antibacterial agent commonly used worldwide as an oral antiseptic mouthwash. ${ }^{37}$ This result showed that the CGF in both forms possessed an antibacterial effect on both microorganisms. Additionally, at $100 \%$ concentration of the CGF, its effectiveness was at $0.12 \%$ of the $\mathrm{CHX}$ in inhibiting the growth of both bacteria. Existing literatures showed that the other platelet concentrate forms such as the PRF and i-PRF showed antibacterial effects against a wide array of microorganisms including the Candida albicans, Porphyromonas gingivalis, and Aggregatibacter actinomycetemcomitans by showing positive zone of inhibition with no available reports on the CGF action as an antibacterial agent. ${ }^{21,38,39}$ Despite the established data on the antibacterial effect, the exact component within the platelet concentrate and mechanism involved for its antibacterial effect are still largely unknown, and the anecdotal and empirical evidence claiming the production of oxygen ion and metabolites, various antibacterial action including aggregation and binding to the microorganisms, the release of host defense antimicrobial peptides, direct interaction of platelets with microorganisms, specific activation of antioxidants, and production of particular proteins such as defensins, lactoferrins, and cathelicidins that are involved in the host-specific immune response were suggested. ${ }^{21,39-42}$

Bacterial biofilms are serious global health issues and have emerged as key factors in antibiotic resistance due to their abilities to tolerate antibiotics, host defense systems, and other external stresses. Therefore, it contributes to persistent chronic infections. Biofilms not only provide the protection for microorganism from altered $\mathrm{pH}$, osmolarity, nutrient scarcity, and mechanical and shear forces, but also have the ability to block the access of bacterial biofilm communities from antibiotics and host's immune cells. Thus, biofilm matrix provides the additional resistance power to bacteria, which makes them to not only be able to tolerate harsh conditions, but also be resistant to antibiotics. ${ }^{43}$ In this study, the results showed that the biofilm formation produced by S. mutans was inhibited more effectively after being treated with the CGF as compared with the control group, notably at the highest concentration. However, the effect of the CGF against the biofilm formation produced by $S$. aureus was comparable to the control group at all concentrations. To the best of our knowledge, this study is the first assessment report on the effect of the CGF against the biofilm formation produced by the pathogenic bacteria. This provides an excellent platform for the CGF utilization in a plethora of clinical chronic conditions and orofacial disease especially associated with $S$. aureus infection, such as facial cellulitis, dentoalveolar abscess, and peri-implant infection. ${ }^{25-28,44}$ Since most of clinical findings established in the body of literature focused on application of CGFas a biomaterial in implant-related treatment and maxillofacial and periodontal regenerative therapy, this preliminary finding will enhance the potential synergistic effects of CGF both as a regenerative biomaterial and as a potential antimicrobial agent in those therapies involved. ${ }^{45-47}$ In implant therapy, various techniques had been advocated to improve the antibacterial effect especially on the surface of the implant and on the implant abutment, namely anodization, nano-modified implant surface, and other methods to maintain a bacteriafree layer on the surface structure and inadvertently aid and enhance the osseointegration process. ${ }^{48,49}$ This preliminary finding will further consolidate the utilization of autologous platelet concentrate especially CGF as a natural biomaterial retrieved from the host, with almost negligible biological and biomechanical risk involved, to aid in potential prevention of bacteria growth and domination and thus promote osseointegration. There was another isolated clinical report on utilization of PRP mouth rinse as a treatment modality on nonresponding lichen planus; however, this is merely a single report and warrants more clinical studies. ${ }^{50}$ Nevertheless, 
more consolidated research is necessary for this potential to be translated into an established clinical practice.

\section{Conclusion}

The result of this study demonstrated that the CGF has the potential as an antimicrobial and antibiofilm agent for both S. aureus and S. mutans isolates. The antimicrobial and antibiofilm capacity of the CGF was comparable to that of the wellestablished antimicrobial mouth rinse (CHX 0.12\%), as reflected by the result. Nonetheless, careful consideration is needed prior to translating this into clinical practice and further in-depth research is needed utilizing in vivo and clinical trial models. A more elaborative investigation on the CGF against other major bacteria groups will be beneficial as well.

Funding

The authors would like to thank Universiti Sains Islam Malaysia for the support of this study through a research grant (PPPI/TB/FPG/17020).

\section{Conflict of Interest}

None declared.

\section{References}

1 Thorn JJ, Sørensen H, Weis-Fogh U, Andersen M. Autologous fibrin glue with growth factors in reconstructive maxillofacial surgery. Int J Oral Maxillofac Surg 2004;33(01):95-100

2 Marx RE. Platelet-rich plasma (PRP): what is PRP and what is not PRP? Implant Dent 2001;10(04):225-228

3 Dohan DM, Choukroun J, Diss A, et al. Platelet-rich fibrin (PRF): a second-generation platelet concentrate. Part I: technological concepts and evolution. Oral Surg Oral Med Oral Pathol Oral Radiol Endod 2006;101(03):e37-e44

4 Kawase T, Mubarak S, Mourão CF. The platelet concentrates therapy: from the biased past to the anticipated future. Bioengineering (Basel) 2020;7(03):82

5 Prakash S, Thakur A. Platelet concentrates: past, present and future. J Maxillofac Oral Surg 2011;10(01):45-49

6 Rodella LF, Favero G, Boninsegna R, et al. Growth factors, CD34 positive cells, and fibrin network analysis in concentrated growth factors fraction. Microsc Res Tech 2011;74(08):772-777

7 Lokwani BV, Gupta D, Agrawal RS, Mehta S, Nirmal NJ. The use of concentrated growth factor in dental implantology: a systematic review. J Indian Prosthodont Soc 2020;20(01):3-10

8 Kamal A, Salman B, Razak NHA, Samsudin ABR. A comparative clinical study between concentrated growth factor and low-level laser therapy in the management of dry socket. Eur J Dent 2020;14 (04):613-620

9 Yüce MO, Adalı E, Işık G. The effect of concentrated growth factor (CGF) in the surgical treatment of medication-related osteonecrosis of the jaw (MRONJ) in osteoporosis patients: a randomized controlled study. Clin Oral Investig 2021;25(07):4529-4541

10 Mijiritsky E, Assaf HD, Peleg O, Shacham M, Cerroni L, Mangani L. Use of PRP, PRF and CGF in periodontal regeneration and facial rejuvenation-a narrative review. Biology (Basel) 2021;10(04):317

11 Adalı E, Yüce MO, Günbay T, Günbay S. Does concentrated growth factor used with allografts in maxillary sinus lifting have adjunctive benefits? J Oral Maxillofac Surg 2021;79(01):98-108

12 Alauddin MS, Ramli H. Management of membrane exposure utilizing concentrated growth factor (CFG) in guided bone regeneration: a clinical report. Open Dent J 2020;14(01):763-768
13 Pirpir C, Yilmaz O, Candirli C, Balaban E. Evaluation of effectiveness of concentrated growth factor on osseointegration. Int J Implant Dent 2017;3(01):7

14 Yumoto H, Hirota K, Hirao K, et al. The pathogenic factors from oral streptococci for systemic diseases. Int J Mol Sci 2019;20(18): 4571

15 McCormack MG, Smith AJ, Akram AN, Jackson M, Robertson D, Edwards G. Staphylococcus aureus and the oral cavity: an overlooked source of carriage and infection? Am J Infect Control 2015; 43(01):35-37

16 Koukos G, Sakellari D, Arsenakis M, Tsalikis L, Slini T, Konstantinidis A. Prevalence of Staphylococcus aureus and methicillin resistant Staphylococcus aureus (MRSA) in the oral cavity. Arch Oral Biol 2015;60(09):1410-1415

17 Wang H, Ren D. Controlling Streptococcus mutans and Staphylococcus aureus biofilms with direct current and chlorhexidine. AMB Express 2017;7(01):204

18 Feng M, Wang Y, Zhang P, et al. Antibacterial effects of plateletrich fibrin produced by horizontal centrifugation. Int J Oral Sci 2020;12(01):32

19 Varshney S, Dwivedi A, Pandey V. Antimicrobial effects of various platelet rich concentrates-vibes from in-vitro studies-a systematic review. J Oral Biol Craniofac Res 2019;9(04):299-305

20 Jasmine S, A T,Janarthanan K, Krishnamoorthy R, Alshatwi AA. Antimicrobial and antibiofilm potential of injectable platelet rich fibrin-a second-generation platelet concentrate-against biofilm producing oral staphylococcus isolates. Saudi J Biol Sci 2020;27 (01):41-46

21 Kour P, Pudakalkatti PS, Vas AM, Das S, Padmanabhan S. Comparative evaluation of antimicrobial efficacy of platelet-rich plasma, platelet-rich fibrin, and injectable platelet-rich fibrin on the standard strains of Porphyromonas gingivalis and Aggregatibacter actinomycetemcomitans. Contemp Clin Dent 2018;9 (Suppl 2):S325-S330

22 PR708. The antimicrobial activity of PRP, PRF and CGF - a review. J Clin Periodontol 2018;45:363-364

23 Gurenlian JR. The role of dental plaque biofilm in oral health. Am Dental Hygienists' 2007;81(Suppl 5):116

24 Wang Y, Shen X, Ma S, et al. Oral biofilm elimination by combining iron-based nanozymes and hydrogen peroxide-producing bacteria. Biomater Sci 2020;8(09):2447-2458

25 Irani S. Orofacial bacterial infectious diseases: an update. J Int Soc Prev Community Dent 2017;7(Suppl 2):S61-S67

26 Bahl R, Sandhu S, Singh K, Sahai N, Gupta M. Odontogenic infections: Microbiology and management. Contemp Clin Dent 2014;5(03):307-311

27 Murakami M, Nishi Y, Seto K, Kamashita Y, Nagaoka E. Dry mouth and denture plaque microflora in complete denture and palatal obturator prosthesis wearers. Gerodontology 2015;32(03): 188-194

28 Oza N, Doshi JJ. Angular cheilitis: a clinical and microbial study. Indian J Dent Res 2017;28(06):661-665

29 Līduma I, Tračevska T, Bērs U, Žileviča A. Phenotypic and genetic analysis of biofilm formation by Staphylococcus epidermidis. Medicina (Kaunas) 2012;48(06):305-309

30 Faden A. Methicillin-resistant Staphylococcus aureus (MRSA) screening of hospital dental clinic surfaces. Saudi J Biol Sci 2019;26(07):1795-1798

31 Salmanov AG, Bondar TP, Shkorbotun YV, et al. Prevalence of nasal carriage of methicillin-resistant Staphylococcus aureus among healthcare workers in the departments of otorinolaryngology and dentistry in Kyiv, Ukraine. Wiad Lek 2020;73(12 cz 1):2563-2567

32 Vaid T, Kumar S, Mehta R, et al. Clinical and radiographic evaluation of demineralized freeze-dried bone allograft with concentrated growth factor versus concentrated growth factor alone in the treatment of intrabony defects. Med Pharm Rep 2021;94(02): $220-228$ 
33 Korkmaz B, Balli U. Clinical evaluation of the treatment of multiple gingival recessions with connective tissue graft or concentrated growth factor using tunnel technique: a randomized controlled clinical trial. Clin Oral Investig 2021;25(11): 6347-6356

34 Anitua E. The use of plasma-rich growth factors (PRGF) in oral surgery. Pract Proced Aesthet Dent 2001;13(06):487-493, quiz 487-493

35 Bernardi S, Mummolo S, Tecco S, Continenza MA, Marzo G. Histological characterization of Sacco's concentrated growth factors membrane. Int J Morphol 2017;35(01):114-119

36 Miron RJ, Zhang Y. Autologous liquid platelet rich fibrin: a novel drug delivery system. Acta Biomater 2018;75:35-51

37 Brookes ZLS, Bescos R, Belfield LA, Ali K, Roberts A. Current uses of chlorhexidine for management of oral disease: a narrative review. J Dent 2020;103:103497

38 Nagaraja S, Mathew S, Jain N, et al. Study of antibacterial and antifungal efficacy of platelet-rich fibrin and platelet-rich fibrin matrix. J Conserv Dent 2019;22(05):415-419

39 Karde PA, Sethi KS, Mahale SA, Khedkar SU, Patil AG, Joshi CP. Comparative evaluation of platelet count and antimicrobial efficacy of injectable platelet-rich fibrin with other platelet concentrates: an in vitro study. J Indian Soc Periodontol 2017;21(02): 97-101

40 Yeaman MR. The role of platelets in antimicrobial host defense. Clin Infect Dis 1997;25(05):951-968

41 Anitua E, Andia I, Ardanza B, Nurden P, Nurden AT. Autologous platelets as a source of proteins for healing and tissue regeneration. Thromb Haemost 2004;91(01):4-15
42 Intravia J, Allen DA, Durant TJ, et al. In vitro evaluation of the antibacterial effect of two preparations of platelet rich plasma compared with cefazolin and whole blood. Muscles Ligaments Tendons J 2014;4(01):79-84

43 Larsen T, Fiehn NE. Dental biofilm infections - an update. APMIS 2017;125(04):376-384

44 Persson GR, Renvert S. Cluster of bacteria associated with periimplantitis. Clin Implant Dent Relat Res 2014;16(06):783-793

45 Chen H, Su Z, Tian J. “Zipper” concept for designing expanded forehead flap in facial-cervical reconstruction. J Craniofac Surg 2021;32(04):1316-1321

46 Caruana A, Savina D, Macedo JP, Soares SC. From platelet-rich plasma to advanced platelet-rich fibrin: biological achievements and clinical advances in modern surgery. Eur J Dent 2019;13(02): 280-286

47 Sousa F, Machado V, Botelho J, Proença L, Mendes JJ, Alves R. Effect of A-PRF application on palatal wound healing after free gingival graft harvesting: a prospective randomized study. Eur J Dent 2020;14(01):63-69

48 Hosseinpour S, Nanda A, Walsh LJ, Xu C. Microbial decontamination and antibacterial activity of nanostructured titanium dental implants: a narrative review. Nanomaterials (Basel) 2021;11(09): 2336

49 Alauddin MS, Baharuddin AS, Mohd Ghazali MI. The modern and digital transformation of oral health care: a mini review. Healthcare (Basel) 2021;9(02):118

50 Merigo E, Oppici A, Parlatore A, et al. Platelet-rich plasma (PRP) rinses for the treatment of non-responding oral lichen planus: a case report. Biomedicines 2018;6(01):15 